\title{
IMA Commission on New Minerals, Nomenclature and Classification (CNMNC)
}

\section{Newsletter 62}

\author{
Ritsuro Miyawaki (Chairman, CNMNC)1, Frédéric Hatert (Vice-Chairman, CNMNC)², Marco Pasero (Vice-Chairman, \\ CNMNC) $)^{3 *}$ and Stuart J. Mills (Secretary, CNMNC) ${ }^{4}$ \\ ${ }^{1}$ Department of Geology and Paleontology, National Museum of Nature and Science, 4-1-1 Amakubo, Tsukuba 305-0005, Japan - miyawaki@kahaku.go.jp; \\ ${ }^{2}$ Laboratoire de Minéralogie, Université de Liège, B-4000 Liège, Belgium - fhatert@uliege.be; ${ }^{3}$ Dipartimento di Scienze della Terra, Università di Pisa, Via Santa Maria \\ 53, I-56126 Pisa, Italy - marco.pasero@unipi.it; and ${ }^{4}$ Geosciences, Museums Victoria, PO Box 666, Melbourne, Victoria 3001, Australia - smills@museum.vic.gov.au
}

The information given here is provided by the IMA Commission on New Minerals, Nomenclature and Classification for comparative purposes and as a service to mineralogists working on new species.

Each mineral is described in the following format:

Mineral name, if the authors agree on its release prior to the full description appearing in press

Chemical formula

Type locality

Full authorship of proposal

E-mail address of corresponding author

Relationship to other minerals

Crystal system, Space group; Structure determined, yes or no

Unit-cell parameters

Strongest lines in the powder X-ray diffraction pattern

Type specimen repository and specimen number

Citation details for the mineral prior to publication of full description

Citation details concern the fact that this information will be published in the Mineralogical Magazine on a routine basis, as well as being added month by month to the Commission's web site.

It is still a requirement for the authors to publish a full description of the new mineral.

NO OTHER INFORMATION WILL BE RELEASED BY THE COMMISSION

\section{NEW MINERAL PROPOSALS APPROVED IN JUNE 2021}

IMA No. 2021-008

Gachingite

$\mathrm{Au}\left(\mathrm{Te}_{1-x} \mathrm{Se}_{x}\right) 0.2 \approx x \leq 0.5$

Gaching ore occurrence, Maletoyvayam ore field, southwestern part of the Koryak Highland, central Kamchatka volcanic belt, Far Eastern Region, Russia (60¹9’51.87” N, 16446'25.65” E) Nadhezda D. Tolstykh, Marek Tuhý*, Anna Vymazalová, Jakub Plášil, František Laufek, Filip Košek and Evgeny G. Sidorov

*E-mail: marek.tuhy@geology.cz

Known synthetic analogue

Orthorhombic: Cmce

$a=7.54, b=5.74, c=8.90 \AA$
4.462(41), 3.194(27), 2.884(74), 2.878(69), 2.734(100), 2.231(28), 2.215(43), $1.813(62)$

Type material is deposited in the mineralogical collections of the Central Siberian Geological Museum (CSGM), V.S. Sobolev Institute of Geology and Mineralogy, Siberian Branch of the Russian Academy of Science, prosp. Akad. Koptyuga 3, 630090 Novosibirsk, Russia, catalogue number V-10/1

How to cite: Tolstykh, N.D., Tuhý, M., Vymazalová, A., Plášil, J., Laufek, F., Košek, F. and Sidorov, E.G. (2021) Gachingite, IMA 2021-008. CNMNC Newsletter 62; Mineralogical Magazine, 85, https://doi.org/10.1180/mgm.2021.62

IMA No. 2021-013

Calciohatertite

$\mathrm{NaNaCa}\left(\mathrm{CaFe}^{3+}\right)\left(\mathrm{AsO}_{4}\right)_{3}$

Arsenatnaya fumarole, second scoria cone of the Northern Breakthrough of the Great Tolbachik fissure eruption, Tolbachik volcano, Kamchatka, Far-Eastern Region, Russia $\left(55^{\circ} 41^{\prime} \mathrm{N}, 160^{\circ} 14^{\prime} \mathrm{E}, 1200 \mathrm{~m}\right.$ a.s.l.) 
Natalia N. Koshlyakova*, Igor V. Pekov, Dmitry I. Belakovskiy, Marina F. Vigasina, Natalia V. Zubkova, Atali A. Agakhanov, Sergey N. Britvin, Evgeny G. Sidorov and Dmitry Y. Pushcharovsky

*E-mail: nkoshlyakova@gmail.com

Alluaudite supergroup

Monoclinic: $C 2 / c$; structure determined

$a=12.6092(8), b=13.2434(5), c=6.8096(3) \AA, \beta=114.554(7)^{\circ}$

6.64(11), 3.714(16), 3.252(15), 3.093(14), 3.066(12), 2.922(17), 2.885(100), 2.665(25)

Type material is deposited in the collections of the Fersman Mineralogical Museum, Russian Academy of Sciences, Leninskiy Prospekt 18-2, Moscow 119071, Russia, registration number 5641/1

How to cite: Koshlyakova, N.N., Pekov, I.V., Belakovskiy, D.I., Vigasina, M.F., Zubkova, N.V., Agakhanov, A.A., Britvin, S.N., Sidorov, E.G. and Pushcharovsky, D.Y. (2021) Calciohatertite, IMA 2021-013. CNMNC Newsletter 62; Mineralogical Magazine, 85, https://doi.org/10.1180/mgm. 2021.62

\section{IMA No. 2021-018}

Tennantite- $(\mathrm{Ni})$

$\mathrm{Cu}_{6}\left(\mathrm{Cu}_{4} \mathrm{Ni}_{2}\right) \mathrm{As}_{4} \mathrm{~S}_{13}$

Luobusa deposit, about $16 \mathrm{~km}$ northeast of Qusum County, Tibet, China (29¹0'58.0” N, 92 $\left.17^{\prime} 47.6^{\prime \prime} \mathrm{E}\right)$

Yanjuan Wang, Rujun Chen, Xiangping $\mathrm{Gu}^{*}$, Zhusen Yang,

Zenqian Hou, Guang Fan, Lijuan Ye and Kai Qu

*E-mail: guxp2004@163.com

Tetrahedrite group

Cubic: $I \overline{4} 3 m$; structure determined

$a=10.2957(9) \AA$

4.233(27), 2.968(100), 2.753(13), 2.563(20), 2.017(13), 1.815(33), $1.549(27)$

Type material is deposited in the mineralogical collections of the Geological Museum of China, No. 16 Yangrou Hutong, Xisi, Beijing 100031, People's Republic of China, catalogue number M16117

How to cite: Wang, Y., Chen, R., Gu, X., Yang, Z., Hou, Z., Fan, G., Ye, L. and Qu, K. (2021) Tennantite-(Ni), IMA 2021-018. CNMNC Newsletter 62; Mineralogical Magazine, 85, https:// doi.org/10.1180/mgm.2021.62

\section{IMA No. 2021-019}

\section{Tomiolloite}

$\mathrm{Al}_{12}\left(\mathrm{Te}^{4+} \mathrm{O}_{3}\right)_{5}\left[\left(\mathrm{SO}_{3}\right)_{0.5}\left(\mathrm{SO}_{4}\right)_{0.5}\right](\mathrm{OH})_{24}$

Bambolla mine (aka Moctezuma mine), Moctezuma, Sonora, Mexico (2941'30” N, 10942'43” W)

Owen P. Missen*, Stuart J. Mills, Mike S. Rumsey, John Spratt, Jens Najorka, Anthony R. Kampf and Brent Thorne

${ }^{\star}$ E-mail: omissen@museum.vic.gov.au

New structure type

Hexagonal: $\mathrm{Pb}_{3} / \mathrm{m}$; structure determined

$a=13.336(2), c=11.604(2) \AA$

11.667(89), 8.240(38), 4.395(29), 4.107(29), 3.503(31), 3.223(100), 2.905(37), 2.593(21)

Type material is deposited in the mineralogical collections of the Museums Victoria, GPO Box 666, Melbourne 3001, Victoria, Australia, specimen number M55489

How to cite: Missen, O.P., Mills, S.J., Rumsey, M.S., Spratt, J., Najorka, J., Kampf, A.R. and Thorne, B. (2021) Tomiolloite,
IMA 2021-019. CNMNC Newsletter 62; Mineralogical Magazine, 85, https://doi.org/10.1180/mgm.2021.62

\section{IMA No. 2021-020}

Shasuite

$\mathrm{CaNi}_{3}\left(\mathrm{P}_{2} \mathrm{O}_{7}\right)_{2}$

Halamish Wadi (Nahal Halamish), Hatrurim basin, Negev desert, Israel ( $\left.31^{\circ} 09^{\prime} 47^{\prime \prime} \mathrm{N}, 35^{\circ} 17^{\prime} 57^{\prime \prime} \mathrm{E}\right)$

Sergey N. Britvin*, Michail N. Murashko, Yevgeny Vapnik, Natalia S. Vlasenko, Oleg S. Vereshchagin, Maria G. Krzhizhanovskaya and Vladimir N. Bocharov

*E-mail: sbritvin@gmail.com

Known synthetic analogue

Monoclinic: $P 2_{1} / c$; structure determined

$a=7.330(2), b=7.573(2), c=9.391(3) \AA, \beta=112.02(3)^{\circ}$

6.796(38), 5.058(32), 3.787(28), 3.070(50), 2.921(100), 2.901(29), 2.648(32), 2.425(28)

Type material is deposited in the collections of the Fersman Mineralogical Museum, Russian Academy of Sciences, Leninskiy Prospekt 18-2, Moscow 119071, Russia, registration number 5630/1

How to cite: Britvin, S.N., Murashko, M.N., Vapnik, Y., Vlasenko, N.S., Vereshchagin, O.S., Krzhizhanovskaya, M.G. and Bocharov, V.N. (2021) Shasuite, IMA 2021-020. CNMNC Newsletter 62; Mineralogical Magazine, 85, https:// doi.org/10.1180/mgm.2021.62

\section{IMA No. 2021-021}

Illoqite-(Ce)

$\mathrm{Na}_{2} \mathrm{NaBaCeZnSi}_{6} \mathrm{O}_{17}$

Taseq Slope, northern part of Ilímaussaq alkaline complex,

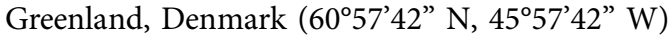

Emil Holtung Gulbransen, Fabrice Dal Bo, Muriel M.L. Erambert and Henrik Friis*

*E-mail: henrik.friis@nhm.uio.no

Nordite group

Orthorhombic: Pcca; structure determined

$a=14.5596(4), b=5.2213(1), c=19.8270(4) \AA$

7.266(79), 4.688(44), 4.241(64), 3.486(79), 3.340(52), 2.986(67), 2.882(100), 2.789(44)

Type material is deposited in the mineralogical collections of the Natural History Museum, University of Oslo, NHM Økern, Kabelgata 38-40, 0580 Oslo, Norway, catalogue numbers KNR 44274 and KNR 44275

How to cite: Gulbransen, E.H., Dal Bo, F., Erambert, M.M.L. and Friis, H. (2021) Illoqite-(Ce), IMA 2021-021. CNMNC Newsletter 62; Mineralogical Magazine, 85, https://doi.org/10. $1180 /$ mgm.2021.62

\section{IMA No. 2021-024}

Paramarkeyite

$\mathrm{Ca}_{2}\left(\mathrm{UO}_{2}\right)\left(\mathrm{CO}_{3}\right)_{3} \cdot 5 \mathrm{H}_{2} \mathrm{O}$

Markey mine, Red Canyon, White Canyon District, San Juan Co., Utah, USA (3732'57” N, $\left.110^{\circ} 18^{\prime} 08^{\prime \prime} \mathrm{W}\right)$

Anthony R. Kampf ${ }^{\star}$, Travis A. Olds, Jakub Plášil, Peter C. Burns, Radek Škoda and Joe Marty

*E-mail: akampf@nhm.org

Chemically and structurally related to markeyite and pseudomarkeyite

Monoclinic: $P 2_{1} / n$; structure determined

$a=17.9507(7), b=18.1030(8), c=18.369(1) \AA, \beta=108.029(8)^{\circ}$ 
8.54(100), 6.31(91), 5.66(45), 5.52(46), 4.759(45), 4.371(97), 3.544(41), 2.858(38)

Type material is deposited in the mineralogical collections of the Natural History Museum of Los Angeles County, 900 Exposition Boulevard, Los Angeles, CA 90007, USA, catalogue numbers 67487 and 67488

How to cite: Kampf, A.R., Olds, T.A., Plášil, J., Burns, P.C., Škoda, R. and Marty, J. (2021) Paramarkeyite, IMA 2021-024. CNMNC Newsletter 62; Mineralogical Magazine, 85, https:// doi.org/10.1180/mgm.2021.62

\section{IMA No. 2021-025}

Zoisite- $(\mathrm{Pb})$

$\mathrm{CaPbAl}_{3}\left[\mathrm{Si}_{2} \mathrm{O}_{7}\right]\left[\mathrm{SiO}_{4}\right] \mathrm{O}(\mathrm{OH})$

Jakobsberg, Filipstad district, Värmland County, Sweden $\left(59^{\circ} 49^{\prime} 40^{\prime \prime} \mathrm{N}, 14^{\circ} 06^{\prime} 25^{\prime \prime} \mathrm{E}\right)$

Natale Perchiazzi ${ }^{\star}$ Knut Eldjarn, Daniela Mauro and Pietro Vignola

*E-mail: natale.perchiazzi@unipi.it

Epidote supergroup

Orthorhombic: Pnma; structure determined

$a=16.3978(6), b=5.5953(2), c=10.1953(3) \AA$

8.63(s), 8.11(mw), 4.895(m), 4.210(m), 3.660(s), 3.097(mw), $2.900(\mathrm{~s}), 2.725(\mathrm{~m})$

Type material is deposited in the mineralogical collections of the Museo di Storia Naturale, Università di Pisa, Via Roma 79, Calci (PI), Italy, catalogue number 19927

How to cite: Perchiazzi, N., Eldjarn, K., Mauro, D. and Vignola, P. (2021) Zoisite-(Pb), IMA 2021-025. CNMNC Newsletter 62; Mineralogical Magazine, 85, https://doi.org/10.1180/mgm.2021. 62

\section{IMA No. 2021-026}

Nabateaite

$\mathrm{Fe}_{2} \mathrm{P}_{2} \mathrm{O}_{7}$

Halamish Wadi (Nahal Halamish), Hatrurim basin, Negev desert, Israel $\left(31^{\circ} 09^{\prime} 47^{\prime \prime} \mathrm{N}, 35^{\circ} 17^{\prime} 57^{\prime \prime} \mathrm{E}\right)$

Sergey N. Britvin*, Michail N. Murashko, Yevgeny Vapnik, Natalia S. Vlasenko, Oleg S. Vereshchagin, Maria G. Krzhizhanovskaya and Vladimir N. Bocharov

${ }^{\star}$ E-mail: sbritvin@gmail.com

Known synthetic analogue

Triclinic: $P \overline{1}$; structure determined

$a=4.488(2), \quad b=5.299(3), \quad c=5.483(3) \quad \AA, \quad \alpha=103.96(4)$,

$\beta=98.28(4), \gamma=98.71(3)^{\circ}$

4.357(19), 3.074(38), 3.056(66), 3.025(100), 2.951(97), 2.627(15), 2.613(22), 2.166(14)

Type material is deposited in the collections of the Fersman Mineralogical Museum, Russian Academy of Sciences, Leninskiy Prospekt 18-2, Moscow 119071, Russia, registration number 5684/1

How to cite: Britvin, S.N., Murashko, M.N., Vapnik, Y., Vlasenko, N.S., Vereshchagin, O.S., Krzhizhanovskaya, M.G. and Bocharov, V.N. (2021) Nabateaite, IMA 2021-026. CNMNC Newsletter 62; Mineralogical Magazine, 85, https:// doi.org/10.1180/mgm.2021.62

\section{IMA No. 2021-027}

Pseudodickthomssenite

$\mathrm{Mg}\left(\mathrm{VO}_{3}\right)_{2} \cdot 8 \mathrm{H}_{2} \mathrm{O}$

Pickett Corral mine, Bull Canyon, Montrose Co., Colorado, USA ( $\left.38^{\circ} 11^{\prime} 58^{\prime \prime} \mathrm{N}, 108^{\circ} 50^{\prime} 24^{\prime \prime} \mathrm{W}\right)$
Anthony R. Kampf*, John M. Hughes, Chi Ma and Joe Marty *E-mail: akampf@nhm.org

Chemically and structurally related to dickthomssenite

Triclinic: $P \overline{1}$; structure determined

$a=7.3566(6), b=9.4672(9), c=9.5529(9) \AA, \alpha=104.205(7)$,

$\beta=100.786(7), \gamma=100.157(7)^{\circ}$

8.971(100), 7.494(10), 4.578(15), 4.473(21), 3.502(15), 2.979

(31), 2.906(12), 1.840(12)

Type material is deposited in the mineralogical collections of the Natural History Museum of Los Angeles County, 900 Exposition Boulevard, Los Angeles, CA 90007, USA, catalogue numbers 76141

How to cite: Kampf, A.R., Hughes, J.M., Ma, C. and Marty, J. (2021) Pseudodickthomssenite, IMA 2021-027. CNMNC Newsletter 62; Mineralogical Magazine, 85, https://doi.org/10. $1180 /$ mgm.2021.62

\section{IMA No. 2021-029}

Samraite

$\mathrm{Ni}_{2} \mathrm{P}_{2} \mathrm{O}_{7}$

Halamish Wadi (Nahal Halamish), Hatrurim basin, Negev desert, Israel (31 $\left.09^{\prime} 47^{\prime \prime} \mathrm{N}, 35^{\circ} 17^{\prime} 57^{\prime \prime} \mathrm{E}\right)$

Sergey N. Britvin*, Michail N. Murashko, Oleg S. Vereshchagin, Yevgeny Vapnik, Natalia S. Vlasenko, Maria G. Krzhizhanovskaya and Vladimir N. Bocharov

${ }^{\star}$ E-mail: sbritvin@gmail.com

Known synthetic analogue

Monoclinic: $P 2_{1} / c$; structure determined

$a=4.4640(4), b=9.8966(10), c=5.2133(6) \AA, \beta=97.505(4)^{\circ}$

4.948(10), 4.426(13), 3.013(64), 2.912(100), 2.500(20), 2.115(13), 2.039(17), 1.962(13)

Type material is deposited in the collections of the Fersman Mineralogical Museum, Russian Academy of Sciences, Leninskiy Prospekt 18-2, Moscow 119071, Russia, registration number $5630 / 1$

How to cite: Britvin, S.N., Murashko, M.N., Vereshchagin, O.S., Vapnik, Y., Vlasenko, N.S., Krzhizhanovskaya, M.G. and Bocharov, V.N. (2021) Samraite, IMA 2021-029. CNMNC Newsletter 62; Mineralogical Magazine, 85, https://doi.org/10. $1180 /$ mgm.2021.62

IMA No. 2021-030

Sapozhnikovite

$\mathrm{Na}_{8}\left(\mathrm{Al}_{6} \mathrm{Si}_{6} \mathrm{O}_{24}\right)(\mathrm{HS})_{2}$

Karnasurt Mountain, northern part of the Lovozero alkaline massif, Kola peninsula, Russia (6752'59” N, 34³7’59” E)

Nikita V. Chukanov*, Natalia V. Zubkova, Igor V. Pekov, Roman Y. Shendrik, Dmitry A. Varlamov, Marina F. Vigasina, Dmitry I. Belakovskiy, Sergey N. Britvin, Vasiliy O. Yapaskurt and Dmitry Y. Pushcharovsky

${ }^{\star}$ E-mail: sbritvin@gmail.com

Sodalite group

Cubic: $P \overline{4} 3 n$; structure determined

$a=8.91462(7) \AA$

6.30(37), 3.638(100), 2.821(14), 2.572(18), 2.382(16), 2.101(29), $1.576(8), 1.486(8)$

Type material is deposited in the collections of the Fersman Mineralogical Museum, Russian Academy of Sciences, Leninskiy Prospekt 18-2, Moscow 119071, Russia, registration number 5693/1

How to cite: Chukanov, N.V., Zubkova, N.V., Pekov, I.V., Shendrik, R.Y., Varlamov, D.A., Vigasina, M.F., Belakovskiy, 
D.I., Britvin, S.N., Yapaskurt, V.O. and Pushcharovsky, D.Y. (2021) Sapozhnikovite, IMA 2021-030. CNMNC Newsletter 62; Mineralogical Magazine, 85, https://doi.org/10.1180/mgm. 2021.62

IMA No. 2021-031

Tetrahedrite-(Ni)

$\mathrm{Cu}_{6}\left(\mathrm{Cu}_{4} \mathrm{Ni}_{2}\right) \mathrm{Sb}_{4} \mathrm{~S}_{13}$

Luobusa deposit, about $16 \mathrm{~km}$ northeast of Qusum County,

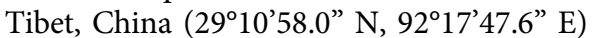

Yanjuan Wang, Rujun Chen, Xiangping $\mathrm{Gu}^{*}$, Zenqian Hou, Zhusen Yang, Guochen Dong, Hu Guo and Kai Qu

*E-mail: guxp2004@163.com

Tetrahedrite group

Cubic: $I \overline{4} 3 m$; structure determined

$a=10.3478(4) \AA$

2.987(100), 3.659(10), 2.766(9), 2.587(21), 2.439(8), 2.029(6),

$1.829(41), 1.560(21)$

Type material is deposited in the mineralogical collections of the Geological Museum of China, No. 16 Yangrou Hutong, Xisi, Beijing 100031, People's Republic of China, catalogue number M16119

How to cite: Wang, Y., Chen, R., Gu, X., Hou, Z., Yang, Z., Dong, G., Guo, H. and Qu, K. (2021) Tetrahedrite-(Ni), IMA 2021-031. CNMNC Newsletter 62; Mineralogical Magazine, 85, https://doi.org/10.1180/mgm.2021.62

\section{NEW MINERAL PROPOSALS APPROVED IN JULY 2021}

IMA No. 2021-032

Qeltite

$\mathrm{Ca}_{3} \mathrm{TiSi}_{2}\left(\mathrm{Fe}_{2}^{3+} \mathrm{Si}\right) \mathrm{O}_{14}$

Nabi Musa, Judean Desert, West Bank, Palestine $\left(31^{\circ} 48^{\prime} \mathrm{N}\right.$, $35^{\circ} 25^{\prime} \mathrm{E}$ )

Irina O. Galuskina*, Marcin Stachowicz, Yevgeny Vapnik, Grzegorz Zeliński, Krzysztof Woźniak and Evgeny Galuskin

*E-mail: irina.galuskina@us.edu.pl

The $\mathrm{Fe}^{3+}$ analogue of paqueite

Trigonal: $P 32_{1}$; structure determined

$a=8.0077(5), c=4.9956(4) \AA$

6.935(31), 4.004(13), 3.124(100), 2.849(61), 2.621(27), 2.321(45), $1.923(13), 1.808(30)$

Type material is deposited in the collections of the Fersman Mineralogical Museum, Russian Academy of Sciences, Leninskiy Prospekt 18-2, Moscow 119071, Russia, registration number $5695 / 1$

How to cite: Galuskina, I.O., Stachowicz, M., Vapnik, Y., Zeliński, G., Woźniak, K. and Galuskin, E. (2021) Qeltite, IMA 2021-032. CNMNC Newsletter 62; Mineralogical Magazine, 85, https://doi.org/10.1180/mgm.2021.62

\section{IMA No. 2021-033}

Sergeysmirnovite

$\mathrm{MgZn}_{2}\left(\mathrm{PO}_{4}\right)_{2} \cdot 4 \mathrm{H}_{2} \mathrm{O}$

Kester deposit, Yana-Adycha Region, Verkhoyansk District, Sakha Republic (Yakutia), Russia

Victor N. Yakovenchuk, Yakov A. Pakhomovsky, Nataliya G. Konoplyova, Taras L. Panikorovskii, Ayya Bazai, Julia A. Mikhailova, Vladimir N. Bocharov and Sergey V. Krivovichev ^E-mail: skrivovi@mail.ru

A dimorph of reaphookhillite
Orthorhombic: Pnma; structure determined

$a=10.6286(4), b=18.3700(6), c=5.0206(2) \AA$

5.28(100), 4.576(33), 3.999(24), 3.877(24), 3.387(22), 3.015(44), 2.854(57), 3.647(34)

Type material is deposited in the collections of the Mineralogical Museum of the St. Petersburg State University, 7-9 Universitetskaya nab., St. Petersburg 199034, Russia, catalogue no. 19659/1

How to cite: Yakovenchuk, V.N., Pakhomovsky, Y.A., Konoplyova, N.G., Panikorovskii, T.L., Bazai, A., Mikhailova, J.A., Bocharov, V.N. and Krivovichev, S.V. (2021) Sergeysmirnovite, IMA 2021-033. CNMNC Newsletter 62; Mineralogical Magazine, 85, https://doi.org/10.1180/mgm.2021.62

IMA No. 2021-034

Goldhillite

$\mathrm{Cu}_{5} \mathrm{Zn}\left(\mathrm{AsO}_{4}\right)_{2}(\mathrm{OH})_{6} \cdot \mathrm{H}_{2} \mathrm{O}$

Middle Pit, Gold Hill Mine, Tooele Co., Utah, USA $\left(40^{\circ} 26^{\prime} 55^{\prime \prime} \mathrm{N}, 113^{\circ} 07^{\prime} 52^{\prime \prime} \mathrm{W}\right)$

Rezeda M. Ismagilova, Anthony R. Kampf, Elena S. Zhitova, Andrey A. Zolotarev, Justyna Ciesielczuk, Julia A. Mikhailova, Dmitry I. Belakovsky, Vladimir N. Bocharov, Vladimir V. Shilovskikh, Natalia S. Vlasenko, Barbara P. Nash and Sergey V. Krivovichev ${ }^{\star}$

${ }^{\star}$ E-mail: skrivovi@mail.ru

The As analogue of kipushite

Monoclinic: $P 2_{1} / c$; structure determined

$a=12.3573(5), b=9.2325(3), c=10.7163(4) \AA, \beta=97.346(4)^{\circ}$

6.29(10), 4.32(17), 4.09(28), 3.41(23), 2.57(100), 2.17(18), 1.95(22), $1.54(20)$

Type material is deposited in the collections of the Fersman Mineralogical Museum, Russian Academy of Sciences, Leninskiy Prospekt 18-2, Moscow 119071, Russia, registration number 88338 (holotype), and the Natural History Museum of Los Angeles County, 900 Exposition Boulevard, Los Angeles, CA 90007, USA, catalogue number 76142 (cotype) How to cite: Ismagilova, R.M., Kampf, A.R., Zhitova, E.S., Zolotarev, A.A., Ciesielczuk, J., Mikhailova, J.A., Belakovsky, D.I., Bocharov, V.N., Shilovskikh, V.V., Vlasenko, N.S., Nash, B.P. and Krivovichev, S.V. (2021) Goldhillite, IMA 2021-034. CNMNC Newsletter 62; Mineralogical Magazine, 85, https:// doi.org/10.1180/mgm.2021.62

\section{IMA No. 2021-035}

Igelströmite

$\mathrm{Fe}^{3+}(\mathrm{SbPb}) \mathrm{O}_{4}$

Långban deposit, Filipstad district, Värmland, Sweden (59.85 $\mathrm{N}, 14.26^{\circ} \mathrm{E}, 215 \mathrm{~m}$ a.s.l.)

Jörgen Langhof*, Henrik Friis, Andreas Karlsson, Dan Holtstam and Muriel Erambert

${ }^{\star}$ E-mail: jorgen.langhof@nrm.se

Known synthetic analogue

Tetragonal: $P 4_{2} / m b c$; structure determined

$a=8.4856(2), c=6.0450(3) \AA$

$4.25(14), 3.21(100), 3.01(13), 2.687(20), 2.462(22), 1.946(19)$, $1.778(14), 1.667(16)$

Type material is deposited in the mineralogical collections of the Department of Geosciences, Swedish Museum of Natural History, Box 50007, SE-10405 Stockholm, Sweden, collection number GEO-NRM 19255056 (holotype), and the Natural History Museum, University of Oslo, NHM Økern, Kabelgata 
38-40, 0580 Oslo, Norway, catalogue number KNR 44278 (cotype)

How to cite: Langhof, J., Friis, H., Karlsson, A., Holtstam, D. and Erambert, M. (2021) Igelströmite, IMA 2021-035. CNMNC Newsletter 62; Mineralogical Magazine, 85, https:// doi.org/10.1180/mgm.2021.62

\section{IMA No. 2021-037}

Betzite

$\mathrm{Na}_{6} \mathrm{Ca}_{2}\left(\mathrm{Al}_{6} \mathrm{Si}_{6} \mathrm{O}_{24}\right) \mathrm{Cl}_{4}$

Bellerberg paleovolcano, between Mayen and Kottenheim, Laacher See area, Eastern Eifel region, Rhineland-Palatinate, Germany

Nikita V. Chukanov*, Natalia V. Zubkova, Olga N. Kazheva, Dmitry A. Varlamov, Igor V. Pekov, Dmitry I. Belakovskiy, Bernd Ternes, Willi Schüller, Sergey N. Britvin and Dmitry Y. Pushcharovsky
*E-mail: nikchukanov@yandex.ru

Cancrinite group

Hexagonal: $P 6_{3}$; structure determined

$a=12.8166(9), c=5.3562(3) \AA$

11.14(31), 4.833(93), 3.715(95), 3.313(100), 2.787(37), 2.681(56),

2.474(35), 2.146(24)

Type material is deposited in the collections of the Fersman Mineralogical Museum, Russian Academy of Sciences, Leninskiy Prospekt 18-2, Moscow 119071, Russia, registration number 5706/1

How to cite: Chukanov, N.V., Zubkova, N.V., Kazheva, O.N., Varlamov, D.A., Pekov, I.V., Belakovskiy, D.I., Ternes, B., Schüller, W., Britvin, S.N. and Pushcharovsky, D.Y. (2021) Betzite, IMA 2021-037. CNMNC Newsletter 62; Mineralogical Magazine, 85, https://doi.org/10.1180/mgm. 2021.62 